\title{
O NON CUMPRIMENTO DA LEI DE GIBRAT EN ECONOMÍAS EN DESENVOLVEMENTO: O CASO DE ECUADOR NO PERÍODO 2000-2013
}

\author{
Luis Enrique SIMBAÑA-TAIPE, \\ María Jesús RODRÍGUEZ-GULÍAS, \\ David RODEIRO-PAZOS
}

Resumo: Ecuador foi un dos países latinoamericanos que experimentou cambios significativos no campo social e económico nas últimas décadas, converténdose nunha das economías máis importantes da rexión. Por iso, resulta relevante coñecer as relacións existentes entre o tamaño e a taxa de crecemento das empresas deste país. Este traballo realiza a súa achega ao contrastar a lei de Gibrat cunha mostra de 25.179 empresas de todos os sectores económicos para o período 2000-2013. Aplicamos o método xeneralizado dos momentos en dous modelos de crecemento e utilízase como medida de crecemento as vendas e o emprego. As descubertas obtidas indican que as taxas de crecemento das empresas grandes son menores cás das pequenas. Ademais, a idade ten unha relación non linear, xa que namentres que nos seus primeiros anos de vida a relación é negativa, a determinada idade cambia a positiva. Esta investigación achega evidencias para a orientación da política pública en países que se atopan en desenvolvemento.

Palabras chave: tamaño, taxa de crecemento empresarial, idade, endebedamento, Ecuador. Abstract: Ecuador is of the Latin American countries experiencing significant changes in the social and economic field in recent decades, becoming one of the most important economies in the region. Therefore, it is relevant to know the existing relationships between the size and growth of companies in this country. This study accomplishes this by contrasting Gibrat's law in a sample of 25,179 companies from all economic sectors for the period 2000-2013. We apply the generalized method of moments in two growth models and it is used as a measure of growth regarding both sales and employment. Findings show that the growth rates of large companies are lower than those of small companies. In addition, age has a non-linear relationship, while in the first years of life the relationship is negative, at a certain age changes to positive. This research provides evidence for the orientation of public policies in developing countries.

Keywords: size, firm growth rate, age, debt, Ecuador

\section{Introdución}

A República do Ecuador é un país latinoamericano que se atopa en desenvolvemento, a súa economía presenta grandes avances e é considerada a octava economía de América Latina se se ten en conta o produto interno bruto (Arévalo Luna, 2014). No período 20062014 reduciu a pobreza en 12 puntos porcentuais de 38,3\% a 25,8\%, e este logro de política social, xunto con outros indicadores, provocou avances importantes no campo social e económico (Atuesta et al., 2014).

A meirande parte do tecido industrial de Ecuador e, en xeral, de América Latina e o Caribe, está composto por pemes, que veñen sendo o 99\% das empresas (CEPAL, 2015) e representan o $60 \%$ do emprego.

En Ecuador, dos empregados rexistrados na seguridade social en 2014 as microempresas concentran o $24,0 \%$ dos traballadores, as pemes o $34,3 \%$ e as grandes empresas o $41,2 \%$, do que se pode concluír que as pemes están xerando un terzo do emprego no Ecuador (Neira Burneo, 2016). Nesta liña, a experiencia internacional evidenciou o potencial das empresas de menor tamaño para mellorar as condicións de vida de grandes grupos de poboación (Pavón, 2016). 
Coñecer a relación do tamaño das empresas sobre a súa taxa de crecemento foi de grande interese para os investigadores que normalmente a utilizan para analizar a distribución do tamaño da empresa e a estrutura de mercado (Almsafir et al., 2015). Neste senso, a lei de Gibrat, implica que non existe relación entre tamaño e taxa de crecemento, o que significa que todas as empresas teñen a mesma probabilidade de crecer (Fernholz e Koch, 2016).

Como consecuencia, co propósito de contribuír á discusión, esta investigación ten como obxectivo analizar a relación entre o tamaño e a taxa de crecemento das empresas ecuatorianas no período 2000-2013 mediante o contraste da lei de Gibrat.

As principais achegas do noso traballo con respecto á literatura previa pódense resumir no seguinte: En primeiro lugar, utilizaremos o método xeneralizado dos momentos (GMM) co propósito de remediar os problemas de endoxeneidade, efectos fixos non observados e a persistencia, e así obter estimacións consistentes e eficientes. En segundo lugar, empregamos dous modelos de crecemento, a través das vendas e o número de empregados, co fin de obter resultados robustos da sustentabilidade ou non da lei de Gibrat. En terceiro lugar, empregamos unha base de datos que considera todas as empresas activas no período de estudo que adicionalmente pertencen a unha economía en desenvolvemento, o que permitirá cubrir o baleiro existente na literatura sobre os países latinoamericanos.

Este artigo estrutúrase do seguinte xeito: na sección 2, realízase unha revisión da literatura. Na sección 3, descríbense os datos e as variables, así como a estratexia de estimación e a especificación do modelo. Na sección 4, preséntanse os resultados da contrastación empírica. Finalmente, na sección 5, detállanse as principais conclusións, as limitacións e as futuras liñas de investigación.

\section{Revisión da literatura}

O crecemento é un dos obxectivos primordiais das empresas, razón pola que numerosos estudos previos se adicaron a contrastar os factores que o afectan. Un dos conceptos de crecemento que espertou grande interese entre os investigadores dende o punto de vista teórico e empírico é a denominada lei de Gibrat ou lei de efectos proporcionais (Harkati e Mohamad, 2016). Esta lei formula que non existe relación entre o tamaño inicial da empresa e a súa taxa de crecemento, pero esta formulación é soamente unha das suxerencias dentro do modelo de Gibrat (Lotti et al. 2003). Esta corrente de investigación permite o estudo deste fenómeno grazas á flexibilidade que ten para incorporar novos factores explicativos, polo que constitúe unha importante ferramenta para a toma de decisións orientadas a promover o desenvolvemento empresarial.

A lei de Gibrat (1931) foi amplamente debatida, debido a que se converteu nun tema relevante na literatura empírica da organización industrial (Fotopoulos e Giotopoulos, 2010). Esta lei establece un axioma que trata de explicar o proceso de crecemento das empresas, que establece que a probabilidade de cambio no tamaño da empresa nun período determinado é igual para todas as empresas nunha industria determinada (Mansfield, 1962).

A lei de Gibrat ou lei de efectos proporcionais suxire tres postulados: (i) a taxa de crecemento das empresas nun período específico é independente do seu tamaño; (ii) a variabilidade da taxa de crecemento das empresas é independente do seu tamaño e (iii) as taxas de crecementos das empresas en dous períodos consecutivos son independentes entre elas (De Haan et al., 2009).

Os resultados obtidos ata o momento sobre as relacións entre o tamaño da empresa e a súa taxa de crecemento case todos suxiren que as empresas grandes teñen una taxa de crecemento menor cá das pequenas empresas, rexeitando, polo tanto, a lei de Gibrat. 
Porén, outros estudos conseguen soster a formulación da independencia entre o tamaño e a taxa de crecemento entre os que podemos mencionar a Del Monte e Papagni (2003) que confirman a lei de Gibrat analizando unha mostra de 500 empresas italianas no período 1989-1997 e que determinan que o tamaño da empresa ten unha tendencia estocástica sobre o crecemento da empresa. Pola súa banda, Fujiwara et al. (2004) ao analizar empresas de 45 países europeos entre o período 1992-2001 determinaron que as taxas de crecemento son independentes do tamaño da empresa. Do mesmo modo, Choi (2010) utilizando unha mostra de 823 empresas do mercado de seguros dos Estados Unidos para o período 1992-2001, está de acordo cos postulados da lei de Gibrat. Pola súa banda, Leitao et al. (2010), utilizando unha mostra de 39 empresas portuguesas para período 1998-2004, probaron o cumprimento da lei de Gibrat. Namentres, Bentzen et al. (2012) analizando unha mostra de 2.935 empresas industriais dinamarquesas para o período 1990-2004 conclúen que a taxa de crecemento das empresas é independente do seu tamaño.

Pola contra, outros estudos non conseguen verificar o cumprimento da lei de Gibrat. Entre eles podemos mencionar a Hoxha (2008) que utilizando unha mostra de 289 empresas dos sectores de produción, comercio e servizos de Kosovo entre 1997-2002 examinan a lei de Gibrat e atopan que as empresas máis pequenas teñen unha taxa de crecemento máis alta cá doutras empresas, como consecuencia, a lei non se sustenta. Por outra banda, Lotti et al. (2009) están de acordo con esta formulación ao analizar 3.285 empresas italianas, e argumentan que se as empresas pequenas crecen máis rápido ao principio, a independencia ente o tamaño e a taxa de crecemento tórnase máis evidente a medida que pasa o tempo. Daunfeldt e Elert (2013), ao analizar 288.757 empresas suecas durante o período 1998-2004, determinan que as pequenas empresas presentan maior taxa de crecemento cás empresas grandes. Ademais, establecen que existen maior probabilidade de rexeitamento cando a industria se caracteriza por un alto tamaño eficiente medio (MES polas súas siglas en inglés). Neste senso, Nassar et al. (2014), analizando 31.984 empresas de servizos xordanas no período 2009-2011, atopan que as pequenas empresas crecen a taxas máis rápidas cás grandes. Pola súa banda, Mukhopadhyay e AmirKhalkhali (2010), ao analizar unha mostra de 500 empresas grandes dos Estados Unidos para o período 2000-2007, atopan que estas empresas teñen unha taxa de crecemento maior cás outras e, como consecuencia, non se sustenta a lei de Gibrat.

Por último, outros autores en base ás mostras ou aos indicadores que permiten medir o crecemento das empresas, obtiveron resultados parciais sobre os postulados de Gibrat entre os que podemos mencionar a Aslan (2008) ao investigar unha mostra de 500 empresas grandes de Turquía para o período 1985-2004. Este autor determinou que para algunhas industrias, a lei de Gibrat foi aceptada namentres que para outras non. Piergiovanni (2010) non confirma a lei de Gibrat para as primeiras etapas do ciclo de vida de 41.859 empresas italianas no período 1995-2005, determinando que as empresas máis novas crecen máis rápido cás xa establecidas, pero a lei de Gibrat cumprése para as empresas que acadan un limiar determinado en termos de idade. Fotopulos e Giotopoulos (2010) rexeitan a lei de Gibrat utilizando unha mostra de 3.685 empresas gregas entre micro, pequenas e novas para o período 1995-2001, porén, establecen que os patróns de crecemento das empresas maduras seguen un camiño aleatorio. Pola súa banda, Serrasqueiro e Nunes (2010) ao analizar 370 empresas portuguesas no período 19992005, utilizando regresión por cuantís conclúe que a lei de Gibrat é aceptada para os niveis inferiores da distribución de crecemento, porén, para os cuantís superiores é rexeitada. Tang (2015), ao investigar unha mostra de 2185 empresas do sector enerxético sueco durante o período 1997-2011, determina que a lei de Gibrat non se cumpre a curto prazo, pero se sustenta a longo prazo, cando as empresas acadan un estado estable. 
A Táboa 1 resume os estudos máis relevantes neste eido, así como as súas principais características.

Táboa 1: Estudos sobre a relación do tamaño sobre a taxa de crecemento da empresa

\begin{tabular}{|c|c|c|c|c|c|}
\hline Autores & País & Período & $\begin{array}{c}\text { Mostra } \\
\text { (empresa } \\
\text { s) }\end{array}$ & $\begin{array}{l}\text { Resultado } \\
\text { Lei de } \\
\text { Gibrat }\end{array}$ & $\begin{array}{l}\text { Medida de } \\
\text { Crecemento }\end{array}$ \\
\hline $\begin{array}{l}\text { Del Monte e Papagni } \\
(2003)\end{array}$ & Italia & 1989-1997 & 500 & Cumpre & Vendas \\
\hline Fujiwara et al. (2004) & $\begin{array}{l}45 \text { países } \\
\text { europeos }\end{array}$ & $1992-2001$ & 260.000 & Cumpre & $\begin{array}{c}\text { Total activos } \\
\text { e №. } \\
\text { empregados }\end{array}$ \\
\hline Choi (2010) & $\begin{array}{l}\text { Estados } \\
\text { Unidos }\end{array}$ & $1992-2001$ & 823 & Cumpre & $\begin{array}{c}\text { №. } \\
\text { empregados }\end{array}$ \\
\hline Leitao et al. (2010) & Portugal & 1998-2004 & 39 & Cumpre & $\begin{array}{l}\text { Total dos } \\
\text { activos }\end{array}$ \\
\hline Bentzen et al. (2012) & Dinamarca & 1990-2004 & 2.935 & Cumpre & $\begin{array}{l}\text { Volume de } \\
\text { vendas }\end{array}$ \\
\hline Hoxha (2008) & Kosovo & 1997-2002 & 289 & $\begin{array}{c}\text { Non } \\
\text { Cumpre }\end{array}$ & $\begin{array}{c}\text { №. } \\
\text { empregados }\end{array}$ \\
\hline Lotti et al. (2009) & Italia & 1987-1994 & 3.285 & $\begin{array}{c}\text { Non } \\
\text { Cumpre }\end{array}$ & $\begin{array}{c}\text { №. } \\
\text { empregados }\end{array}$ \\
\hline $\begin{array}{l}\text { Mukhopadhyay e } \\
\text { AmirKhalkhali (2010) }\end{array}$ & $\begin{array}{l}\text { Estados } \\
\text { Unidos }\end{array}$ & $2000-2007$ & 500 & $\begin{array}{c}\text { Non } \\
\text { Cumpre }\end{array}$ & vendas \\
\hline $\begin{array}{l}\text { Daunfelt e Elert } \\
(2013)\end{array}$ & Suecia & 1998-2004 & 288.757 & $\begin{array}{l}\text { Non } \\
\text { Cumpre }\end{array}$ & $\begin{array}{c}\text { №. } \\
\text { empregados } \\
\text { e ingresos }\end{array}$ \\
\hline Nassar et al. (2014) & Xordania & 2009-2011 & 31.984 & $\begin{array}{c}\text { Non } \\
\text { Cumpre }\end{array}$ & vendas \\
\hline Aslan (2008) & Turquía & 1985-2004 & 500 & Mixtos & Activos netos \\
\hline Piergiovanni (2010) & Italia & $1995-2005$ & 41.859 & Mixtos & $\begin{array}{c}\text { №. } \\
\text { empregados }\end{array}$ \\
\hline $\begin{array}{l}\text { Fotopulos e } \\
\text { Giotopoulos (2010) }\end{array}$ & Grecia & $1995-2001$ & 3.685 & Mixtos & $\begin{array}{l}\text { Total dos } \\
\text { activos }\end{array}$ \\
\hline $\begin{array}{l}\text { Serrasqueiro e Nunes } \\
(2010)\end{array}$ & Portugal & 1999-2005 & 370 & Mixtos & $\begin{array}{c}\text { Total dos } \\
\text { activos }\end{array}$ \\
\hline Tang (2015) & Suecia & $1997-2011$ & 2.185 & Mixtos & $\begin{array}{c}\text { №. } \\
\text { empregados }\end{array}$ \\
\hline
\end{tabular}

Con base no exposto previamente propoñemos a seguinte hipótese:

Hipótese: $O$ tamaño ten un efecto negativo sobre o crecemento das empresas.

0 enfoque desta investigación está pensado en avaliar a relación do tamaño sobre a taxa de crecemento das empresas, co argumento de que as empresas crecen estocasticamente e, polo tanto, todas as industrias tarde ou cedo presentan concentración conforme manifiestan Gibrat (1931); Goddart et al. (2002) e Homma et al. (2014). Porén, dos estudos previos que analizan esta corrente de investigación, pódese destacar que é máis probable que o crecemento tamén estea afectado por diversos factores específicos da industria (Daunfeldt e Elert, 2013).

Por outra banda, nas análises que se realizan sobre o crecemento das empresas, normalmente as investigacións utilizan variables de control, neste traballo tamén se recolleron algunhas das máis habituais, que son expostas a continuación dunha forma máis breve. 
Unha das máis empregadas é a idade, co argumento de que as empresas de maior idade acadarán menor crecemento, considerando que realizar algunha mellora lles resulta máis complexo debido a que posúen maior coñecemento sobre o seu tamaño óptimo (Fariñas e Moreno, 1997; Audretsch et al. 2004; Lotti et al., 2009; Simbaña et al., 2017).

Outro aspecto relevante que consideraron investigacións previas do fenómeno de crecemento é a estratexia de innovación nas empresas, debido a que para acadar a creación de valor no produto ou servizo que as empresas ofrecen no mercado cómpre aplicar novos coñecementos (innovación), razón pola cal se lle considera un determinante chave do crecemento empresarial conforme argumentan Cainelli et al. (2006); Adamou e Sasidharan (2007) e Coad e Rao (2011).

Por outra banda, traballos como os de Stulz (1990) e Santana et al. (2006) prestáronlle atención aos factores financeiros para explicar o crecemento das empresas. 0 argumento principal relaciónase co financiamiento externo que posibilita habilitar o fluxo necesario de caixa e que permite financiar o crecemento das empresas tal e como establecen Serrasqueiro e Nunes (2010); Loi e Khan (2012) e Nunes et al. (2013).

As empresas, co propósito de buscar un mellor posicionamento e ser máis eficientes, vense obrigadas a introducir a tecnoloxía para conseguir crecer e sobrevivir nun mundo cada vez máis competitivo (Pagano e Schivardi, 2003; Daza Izquierdo, 2016). Este argumento serviu aos investigadores para incluila como variable explicativa do crecemento da empresa, atopando xeralmente un impacto positivo sobre a variable de crecemento (Zahra et al., 2007; García-Manjón e Romero-Merino, 2012; Rodríguez-Gulías et al. (2016).

\section{Metodoloxía}

Nesta sección, describimos a mostra e as variables utilizadas, así como a estratexia de estimación e a especificación econométrica do modelo utilizado.

\subsection{Mostra e datos}

A base de datos de partida foi facilitada pola Superintendencia de Compañías, Valores e Seguros de Ecuador e constitúese coas empresas que presentan a información financeira no período 2000-2013.

Táboa 2. Criterio de filtración dos datos da base orixinal

\begin{tabular}{|l|c|c|}
\hline Criterios & $\begin{array}{c}\text { № } \\
\text { empresas } \\
\text { eliminadas }\end{array}$ & № empresas na mostra \\
\hline Base de datos inicial & & 99.872 \\
\hline $\begin{array}{l}\text { Empresas que non cumpren cos } \\
\text { requisitos de formación xurídica }\end{array}$ & 58.539 & 41.333 \\
\hline $\begin{array}{l}\text { Valores que non corresponden á } \\
\text { natureza da conta }\end{array}$ & 16.154 & 25.179 \\
\hline Base de datos final & & 25.179 \\
\hline
\end{tabular}

A mostra final de estudo cobre todas as empresas cuxa formación xurídica corresponde a sociedade anónima e sociedade limitada de todos os sectores económicos de Ecuador e está integrada por un panel non equilibrado de 25.179 empresas. O proceso de filtración dividiuse en dúas etapas, a primeira parte correspondeu á eliminación das empresas que non cumprían cos requisitos de formación xurídica analizada e na segunda fase elimináronse as empresas que tiñan valores que non correspondían coa natureza da conta contable, conforme se presenta na Táboa 2. 


\subsection{Definición e medida das variables}

As variables utilizadas na análise empírica preséntanse na Táboa 3, as cales se atopan clasificadas en tres grupos: taxa de crecemento como variable dependente, tamaño como principal variable independente e as características empresariais e financeiras, como variables de control.

Táboa 3: Especificación das variables independentes

\begin{tabular}{|c|c|c|c|c|}
\hline Grupo & Factor & Descrición & Media & $\begin{array}{c}\text { Signo } \\
\text { agardad } \\
0\end{array}$ \\
\hline \multicolumn{5}{|c|}{ VARIABLES DEPENDENTES } \\
\hline \multirow[t]{2}{*}{ Tamaño } & $L N V T_{t}$ & vendas netas & Logaritmo natural das vendas & \\
\hline & $\operatorname{LNEM}_{t}$ & Emprego & Logaritmo natural do emprego & \\
\hline \multirow[t]{2}{*}{ Crecemento } & $G_{-} V T$ & $\begin{array}{l}\text { Taxa de crecemento das } \\
\text { vendas netas }\end{array}$ & $\begin{array}{l}\text { Logaritmo natural } \\
\text { (vendas/vendas } \mathrm{t}-1 \text { ) }\end{array}$ & \\
\hline & $G_{-} E M$ & $\begin{array}{l}\text { Taxa de crecemento do } \\
\text { emprego }\end{array}$ & $\begin{array}{l}\text { Logaritmo natural } \\
\text { (Emprego/Emprego t-1) }\end{array}$ & \\
\hline \multicolumn{5}{|c|}{ VARIABLE INDEPENDENTE Principais } \\
\hline \multirow[t]{2}{*}{ Tamaño } & $L N V T_{(t-1)}$ & $\begin{array}{l}\text { vendas retardadas un } \\
\text { período }\end{array}$ & $\begin{array}{l}\text { Logaritmo natural das vendas } \\
\text { do período previo }\end{array}$ & - \\
\hline & $\operatorname{LNEM}_{(t-1)}$ & $\begin{array}{l}\text { Emprego retardado un } \\
\text { período }\end{array}$ & $\begin{array}{l}\text { Logaritmo natural do emprego } \\
\text { no período previo }\end{array}$ & - \\
\hline \multicolumn{5}{|c|}{ VARIABLES DE CONTROL } \\
\hline \multirow{7}{*}{$\begin{array}{l}\text { Característic } \\
\text { as } \\
\text { empresariais } \\
\text { e financeiras }\end{array}$} & $L N E D A D$ & Idade & Logaritmo natural da idade & - \\
\hline & LNEDADCUAD & Idade ao cadrado & $\begin{array}{l}\text { Logaritmo natural da idade ao } \\
\text { cadrado }\end{array}$ & + \\
\hline & INNOV & Innovación & Activos intanxibles/vendas & + \\
\hline & ALTA_TECH & Tecnoloxía & $\begin{array}{l}1 \text { se pertence á alta tecnoloxía e } \\
0 \text { para as outras }\end{array}$ & + \\
\hline & MEDALT_TECH & Tecnoloxía & $\begin{array}{l}1 \text { se pertence á media alta } \\
\text { tecnoloxía e } 0 \text { para as outras }\end{array}$ & + \\
\hline & MEDBAJA_TECH & Tecnoloxía & $\begin{array}{l}1 \text { se pertence á media baixa } \\
\text { tecnoloxía e } 0 \text { para as outras }\end{array}$ & - \\
\hline & $E N D$ & Endebedamento & Total pasivo/Total activos & + \\
\hline
\end{tabular}

\subsection{Estratexia de estimación e especificación do modelo}

A avaliación dos resultados na literatura económica estimou que a lei de Gibrat usa varias ecuacións, das cales Carrizosa (2007) formula tres en particular que confirman a mesma. Dúas delas serán as contrastadas neste traballo e preséntanse a continuación.

A primeira ecuación utiliza o logaritmo do número de empregados ou as vendas pertencentes á empresa $i$ durante o período $t, \mathbb{Z}\left(S_{\beth i, t}\right)$ depende do logaritmo do período anterior $\llbracket\left(S \sum_{i_{s} t-1}\right)$, o que se pode expresar da seguinte forma:

$$
\llbracket \log \left(S \rrbracket_{i, t}\right)=\alpha+\beta \log S_{i, t-1}+\mu_{i, t}
$$

A lei de Gibrat é aceptada cando o coeficiente de $\beta$ é igual a 1, o que significaría que o crecemento da empresa é independente do tamaño inicial. Se $\beta$ é menor ca 1 , as 
empresas máis pequenas teñen un maior crecemento. Se $\beta$ é maior ca 1 , as grandes empresas crecen máis rápido.

A primeira ecuación, a representación logarítmica orixinal da lei de Gibrat, dá lugar á primeira especificación formulada nesta investigación [MODELO 1], deseñada en liña con Colombelli et al. (2013). Deste xeito, empregouse como variable explicativa o tamaño da empresa medido a través do logaritmo natural das vendas netas (LNVT) e do emprego (LNEM) á que, adicionalmente, se incorporan variables relativas á idade empresarial (LNEDAD e LNEDADCUAD), o nivel de endebedamento (END) e a innovación (INNO). É por iso que a continuación se presentan dúas versións do modelo 1, unha por cada variable dependente analizada:

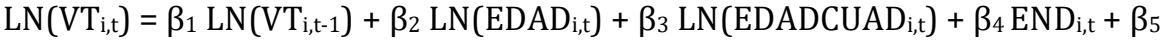

$$
\begin{aligned}
& \left.\mathrm{INNO}_{\mathrm{i}, \mathrm{t}}+\alpha_{\mathrm{i}}+\omega_{\mathrm{i}}+\lambda_{\mathrm{t}}+\varepsilon_{\mathrm{i}, \mathrm{t}} \quad \text { [MODELO } 1 \mathrm{VT}\right] \\
& \operatorname{LN}\left(\mathrm{EM}_{\mathrm{i}, \mathrm{t}}\right)=\beta_{1} \operatorname{LN}\left(\mathrm{EM}_{\mathrm{i}, \mathrm{t}-1)}\right)+\beta_{2} \operatorname{LN}\left(\mathrm{EDAD}_{\mathrm{i}, \mathrm{t}}\right)+\beta_{3} \operatorname{LN}\left(\mathrm{EDADCUAD}_{\mathrm{i}, \mathrm{t}}\right)+\beta_{4} \text { END }_{\mathrm{i}, \mathrm{t}}+\beta_{5} \\
& \text { INNO } \left._{\mathrm{i}, \mathrm{t}}+\alpha_{\mathrm{i}}+\omega_{\mathrm{i}}+\lambda_{\mathrm{t}}+\varepsilon_{\mathrm{i}, \mathrm{t}} \quad \text { [MODELO } 1 \mathrm{EM}\right]
\end{aligned}
$$

Sendo $\mathrm{VT}_{\mathrm{i}, \mathrm{t}}$ e $\mathrm{EM}_{\mathrm{i}, \mathrm{t}}$ as vendas netas e o emprego, respectivamente, da empresa $i$ no período $t$, namentres que $\mathrm{VT}_{\mathrm{i}, \mathrm{t}-1}$ e $\mathrm{EM}_{\mathrm{i}, \mathrm{t}-1}$ fan referencia ao período $t-1$. 0 termo de erro ten varios compoñentes: ademais do efecto individual ou efecto específico de cada empresa $\left(\alpha_{\mathrm{i}}\right), \omega_{\mathrm{i}}$ representa un conxunto de variables dummy relativas ao nivel tecnolóxico (ALTA_TECH; MEDALT_TECH; MEDBAJA_TECH), $\lambda_{\mathrm{t}}$ mide o efecto temporal mediante as correspondentes variables dummy, de forma que se controla o efecto de variables macroeconómicas, e $\varepsilon_{\text {it }}$ é a perturbación aleatoria.

A segunda das ecuacións formuladas por Carrizosa (2007), emprega como variable dependente o incremento do logaritmo de $S_{i t}$. En lugar de obter o tamaño para o seguinte período, obtense o crecemento da empresa durante os períodos $t-\mathbf{1}$ e $t\left(\Delta \llbracket \log \left(S \rrbracket_{i, t}\right)\right) ;$

$$
\Delta \log S_{i, t}=\alpha+\beta \log S_{i, t-1}+\mu_{i, t}
$$

A lei de Gibrat cúmprese se $\beta$ é igual a 0 . Un valor positivo implicaría que as empresas grandes crecerán máis cás empresas máis pequenas, polo que existirá unha diverxencia no tamaño da empresa. Un valor negativo significa que as empresas máis pequenas terán un maior índice de crecemento cás empresas grandes, o que xerará unha converxencia na industria.

Para a segunda ecuación, seguindo a Colombelli et al. (2013), formúlase o MODELO 2 onde se contrasta a relación das taxas anuais de crecemento de vendas e emprego (G_VT o G_EM) e o tamaño empresarial. Para iso, formúlase o modelo 2 coa mesma especificación que o modelo 1, pero cambiando a medida das variables dependentes:

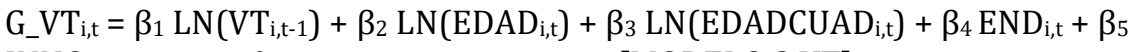

$$
\begin{aligned}
& \text { INNO } \left._{\mathrm{i}, \mathrm{t}}+\alpha_{\mathrm{i}}+\omega_{\mathrm{i}}+\lambda_{\mathrm{t}}+\varepsilon_{\mathrm{i}, \mathrm{t}} \quad \text { [MODELO } 2 \mathrm{VT}\right] \\
& G_{-} E_{i, t}=\beta_{1} \operatorname{LN}\left(E_{i, t-1}\right)+\beta_{2} \operatorname{LN}\left(\operatorname{EDAD}_{\mathrm{i}, \mathrm{t}}\right)+\beta_{3} \operatorname{LN}\left(\operatorname{EDADCUAD}_{\mathrm{i}, \mathrm{t}}\right)+\beta_{4} \operatorname{END}_{\mathrm{i}, \mathrm{t}}+\beta_{5} \\
& \left.\mathrm{INNO}_{\mathrm{i}, \mathrm{t}}+\alpha_{\mathrm{i}}+\omega_{\mathrm{i}}+\lambda_{\mathrm{t}}+\varepsilon_{\mathrm{i}, \mathrm{t}} \quad \text { [MODELO } 2 \mathrm{EM}\right]
\end{aligned}
$$

Onde G_VT $\mathrm{T}_{\mathrm{i}, \mathrm{t}}$ é o crecemento nas vendas netas e $\mathrm{G}_{-} \mathrm{EM}_{\mathrm{i}, \mathrm{t}}$ o crecemento no emprego da compañía i no período $t$, manténdose o resto de variables igual que no modelo 1. 
Estudos previos asumen a existencia de relacións entre o crecemento e o tamaño da empresa, como consecuencia, poden xurdir posiblemente problemas de endoxeneidade. Co propósito de solucionar esta dificultade, utilízanse as variables de tamaño retardadas un período. Polo tanto, o método máis axeitado para xerar estimacións consistentes e eficientes é o método xeneralizado dos momentos (GMM). Os problemas non observados dos efectos fixos das empresas, a persistencia e a endoxeneidade pódense superar usando o sistema GMM, que foi desenvolvido por Arellano e Bond (1991). O sistema GMM é un estimador axeitado para datos de panel no que as variables explicativas non son estritamente exóxenas (Bond, 2002).

\section{Resultados empíricos}

\subsection{Análise univariante}

$\mathrm{Na}$ Táboa 4 preséntanse os estatísticos descritivos das variables dependentes e independentes así como das variables de control no período 2000-2013.

Táboa 4.1: Estatístico descritivo

\begin{tabular}{|c|c|c|c|c|c|c|}
\hline Grupo & Variable & Obs. & Media & $\begin{array}{c}\text { Desviació } \\
\text { n típica. }\end{array}$ & Mín. & Máx. \\
\hline \multirow{2}{*}{ Tamaño } & VT & 204.905 & $\begin{array}{c}3.160 .28 \\
4\end{array}$ & $\begin{array}{c}19.600 .00 \\
0\end{array}$ & 100.002 & $\begin{array}{c}1.750 .000 .00 \\
0\end{array}$ \\
\cline { 2 - 7 } & EM & 152.268 & 32,95 & 156,99 & 0 & 13679 \\
\hline Crecemento & G_VT & 154.330 & 0,12 & 0,50 & $-5,49$ & 7,06 \\
\cline { 2 - 7 } & G_EM & 89.299 & 0,08 & 1,02 & $-8,04$ & 8,35 \\
\hline Idade & EDAD & 204.923 & 12,50 & 10,83 & 1 & 93 \\
\hline Innovación & INNO & 106.771 & 0,01 & 0,05 & 0 & 2,30 \\
\hline Tecnoloxía & TECH & 204.923 & 0,10 & 0,30 & 0 & 1 \\
\hline Apancamento & END & 192.455 & 0,66 & 0,25 & 0 & 1 \\
\hline
\end{tabular}

0 tamaño medio do Valor das Vendas é de 3,16 millóns de Dólares por empresa, cun mínimo de 100 mil e un máximo de 1750 millóns. 0 tamaño medio do número de empregados é de 33, cun mínimo de 0 e un máximo de 13679. Algunhas empresas presentan taxas negativas de crecemento, tanto na variable VT como na variable EM. As taxas de crecemento de VT oscilan entre un mínimo de $-5.49 \%$ e un máximo de $8.35 \%$. As taxas de crecemento de EM oscilan entre un mínimo de -8-04\% e un máximo de 8.35\%.

A táboa 4.2. presenta o crecemento anual medio por tamaño empresarial no período de estudo. A porcentaxe de crecemento das vendas máis elevado deuse nas empresas pequenas, (47.55\%), seguido das medianas $(24.85 \%)$ e das grandes $(16.47 \%)$, pero o incremento total de vendas do conxunto de empresas grandes foi moito maior có das empresas medianas e pequenas.

Táboa 4.2. Crecemento anual medio por tamaño empresarial

\begin{tabular}{|l|c|c|c|}
\hline & \multicolumn{2}{|c|}{ vendas } & Emprego \\
\hline & USD & \% & PERSOAS \\
\hline GRANDE & $4.599 .995,00$ & $16,47 \%$ & 37,25 \\
\hline MEDIANA & $580.115,30$ & $24,85 \%$ & 4,54 \\
\hline PEQUENA & $160.258,40$ & $47,55 \%$ & 1,34 \\
\hline Total & 364.178 & $43,47 \%$ & 2,96 \\
\hline *Cálculos realizados antes da aplicación de logaritmos \\
\hline
\end{tabular}

Estes resultados non significan que as empresas pequenas sexan as que máis contribúen ao incremento do emprego. A contribución ao crecemento do emprego durante o período deste estudo foi a que se indica na Táboa 4.3. 
Tabla 4.3. Participación no incremento do emprego (5) por tamaño empresarial

\begin{tabular}{|c|c|c|c|c|}
\hline & $\begin{array}{c}\text { Incremento medio } \\
\text { do Emprego } \\
\text { (persoas) } \\
(1)\end{array}$ & $\begin{array}{c}\text { Porcentaxe } \\
\text { de empresas } \\
(2)\end{array}$ & $\begin{array}{c}\text { Incremento } \\
\text { medio x } \\
\text { Porcentaxe } \\
(3)=(1) \times(2)\end{array}$ & $\begin{array}{c}\text { Participación } \\
\text { no incremento } \\
(4)\end{array}$ \\
\hline Grandes & 37,25 & 41,2 & 1534,7 & $89,09 \%$ \\
\hline Medianas & 4,54 & 34,3 & 155,7 & $9.04 \%$ \\
\hline Pequenas & 1,34 & 24,0 & $71.032,16$ & $1.87 \%$ \\
\hline Suma & 2,96 & 99,5 & 1722,57 & 100 \\
\hline
\end{tabular}

Nota: Non se inclúen as microempresas. Fonte: elaboración a partir dos datos da Táboa 4.1 para columna (1), e dos datos, xa mencionados, da Seguridade Social para a columna (2).

Conforme a estes datos, o $87.13 \%$ do incremento do emprego, no conxunto de empresas deste estudo, produciuse en empresas grandes, namentres que as medianas aportaron o $8.84 \%$ do devandito incremento e as pequenas o $4.03 \%$. Hai moitas empresas non incluídas no conxunto deste estudo, xa que conforme aos datos do Goberno de Ecuador, o 90\% das empresas son microempresas e non están incluídas. A aportación das grandes empresas ao incremento do emprego é interesante para moitos traballadores, xa que as cifras oficiais indican que o salario medio é máis elevado nesas empresas.

\subsection{Análise multivariante}

Na Táboa 5 recóllense os resultados das estimacións utilizando o método xeneralizado dos momentos, do cal se pode observar que no modelo 1 ao medir o tamaño a través das vendas e os empregados, pódese establecer que as empresas pequenas crecen máis cás grandes. De igual maneira pódese apreciar no modelo 2 ao utilizar como variable dependente a taxa de crecemento das vendas ou o emprego, que as empresas grandes crecen menos cás pequenas.

Táboa 5: Estimacións das vendas netas e o emprego: método xeneralizado dos momentos

\begin{tabular}{|l|c|c|c|c|}
\hline \multirow{2}{*}{} & \multicolumn{2}{|c|}{ Vendas } & \multicolumn{2}{c|}{ Emprego } \\
\cline { 2 - 5 } & MODELO 1 & MODELO 2 & MODELO 1 & MODELO 2 \\
\hline VARIABLE DEPENDENTE $^{2}$ & LNVT & G_VT & LNEM & G_EM \\
\hline & $0,805^{* * *}$ & $-0,195^{* * *}$ & & \\
\hline LNEM $_{(\mathrm{t}-1)}$ ( $)$ & $-0,01$ & $-0,01$ & & \\
\hline & & & $0,477^{* * *}$ & $-0,523^{* * *}$ \\
\hline LNEDAD & & & $-0,028$ & $-0,028$ \\
\hline & $-0,592^{* * *}$ & $-0,592^{* * *}$ & $-0,188^{* *}$ & $-0,188^{* *}$ \\
\hline LNEDADCUAD & $-0,024$ & $-0,024$ & $-0,057$ & $-0,057$ \\
\hline & $0,127^{* * *}$ & $0,127^{* * *}$ & $0,080^{* * *}$ & $0,080^{* * *}$ \\
\hline INNO & $-0,005$ & $-0,005$ & $-0,013$ & $-0,013$ \\
\hline & 0,07 & 0,07 & 0,787 & 0,787 \\
\hline ALTA_TECH & $-0,127$ & $-0,127$ & $-0,413$ & $-0,413$ \\
\hline & $-0,009^{* * *}$ & $-0,009^{* * *}$ & $-0,001$ & $-0,001$ \\
\hline MEDALT_TECH & $-0,002$ & $-0,002$ & $-0,004$ & $-0,004$ \\
\hline & 0,006 & 0,006 & $0,023^{* * *}$ & $0,023^{* * *}$ \\
\hline END & $-0,003$ & $-0,003$ & $-0,006$ & $-0,006$ \\
\hline & $-0,027^{* * *}$ & $-0,027^{* * *}$ & $-0,023$ & $-0,023$ \\
\hline
\end{tabular}




\begin{tabular}{|l|c|c|c|c|}
\hline cons & $3,520^{* * *}$ & $3,520^{* * *}$ & $1,394^{* * *}$ & $1,394^{* * *}$ \\
\hline & $-0,137$ & $-0,137$ & $-0,084$ & $-0,084$ \\
\hline Dummies anos incluídas & Sí & Sí & Sí & Sí \\
\hline № observacións & 78.638 & 78.638 & 25.982 & 25.982 \\
\hline № empresas & 25.179 & 25.179 & 18.817 & 18.817 \\
\hline Instrumentos & 73 & 73 & 55 & 55 \\
\hline Graos de liberdade & 19 & 19 & 13 & 13 \\
\hline Test F & $1.110,60$ & 92,67 & 212,21 & 48,25 \\
\hline F p-val. & 0 & 0 & 0 & 0 \\
\hline Test AR(1) & $-24,68$ & $-24,68$ & $-7,45$ & $-7,45$ \\
\hline AR(1) p-val. & 0 & 0 & 0 & 0 \\
\hline Test AR(2) & 1,24 & 1,24 & 1,45 & 1,45 \\
\hline AR(2) p-val. & 0,216 & 0,216 & 0,148 & 0,148 \\
\hline Estatístico J Hansen & $1.146,80$ & $1.146,80$ & 214,11 & 214,11 \\
\hline J Hansen p-val. & 0 & 0 & 0 & 0 \\
\hline
\end{tabular}

Nota: ${ }^{*} \mathrm{p}<0,05 ;{ }^{* *} \mathrm{p}<0,01 ;{ }^{* * *} \mathrm{p}<0,001$. Os erros estándar corrixidos (Windmeijer, 2005) preséntanse entre paréntesis.

Na primeira versión (modelo 1) para que se cumpra a lei de Gibrat os $\beta_{1=1}$. Os resultados obtidos na Táboa 5 indican que os $\beta_{1<1}$, independentemente de utilizar como variable de crecemento as vendas e o emprego.

Na segunda versión (modelo 2) para que se cumpra a lei de Gibrat, os $\beta_{1=0} 0$. Os resultados obtidos na Táboa 5 indican que os $\beta_{1}<1$, tanto se o crecemento se mide a través das vendas como do emprego.

Os resultados obtidos permiten establecer que as empresas pequenas crecen máis rápido cás grandes e que o crecemento da empresa depende do tamaño da empresa, o que nos permite aceptar a hipótese 1 . Como consecuencia, a lei de Gibrat non se cumpre para as empresas ecuatorianas cuxa formación xurídica é a de sociedade anónima e limitada, os resultados axústanse á evidencia previa sobre o crecemento empresarial obtida por Mukhopadhyay e AmirKhalkhali (2010), Daunfelt e Elert (2013) e Nassar et al. (2014).

A idade da empresa (LNEDAD e LNEDADCUAD) tanto en relación ao tamaño (LNVT e LNEM) como á taxa de crecemento (G_VT e G_EM) evidencia unha relación no linear. Isto significa que a idade nos primeiros anos de vida da empresa exerce un efecto negativo. Porén, a unha determinada idade esta relación cambia e se transforma en positiva, as descobertas axústanse ás atopadas por Rodríguez-Gulías et al. (2016) e Simbaña et al. (2017).

En canto á variable de control relacionada coa innovación (INNO) non se observa ningún efecto significativo sobre o tamaño da empresa (LNVT e LNEM) nin sobre o seu crecemento (G_VT e G_EM) nos dous modelos utilizados.

En referencia á variable relacionada co desempeño en sectores tecnolóxicos das empresas, as estimacións suxiren que realizar actividades en sectores de alta tecnoloxía (ALTA_TECH) exerce un efecto negativo sobre o tamaño e o crecemento medidos a través das vendas. Porén, esta evidencia non se atopa no caso do emprego (LNEM e G_EM). Namentres que ao realizar actividades en sectores de media-alta tecnoloxía, o impacto positivo é significativo, exclusivamente sobre o tamaño e crecemento cando se mide a través do emprego. Estes resultados non se observan no caso das vendas (LNVT e G_VT).

Por último, atópase un efecto negativo significativo do apancamento financeiro (END) sobre o tamaño e o crecemento das empresas medidas a través das vendas, pero os resultados non son significativos para o emprego. Ao testar a especificación dos modelos, o estatístico AR (2), desenvolvido por Arellano e Bond (1991), amosou a ausencia de 
correlación serial de segundo orde nos residuos. Porén, o test J de Hansen indica sobreidentificación para todos os modelos, tanto para vendas como para emprego (Hansen=Prob $>$ Chi2 $<0.05$ ), de forma que se acepta a existencia de correlación entre os instrumentos e o termo de erro, non podendo se confirmar a validez dos instrumentos. Unha posible explicación a este problema é a dimensión temporal da mostra $(\mathrm{T}=14)$. Cando a mostra ten un $\mathrm{T}$ grande $(\mathrm{T}>10)$, o problema de sobreidentificación emerxe facilmente (Labra e Torrecillas, 2014).

Cando $\mathrm{T}$ é grande, o nesgo de panel dinámico faise insignificante e un estimador de efectos fixos máis sinxelo é axeitado (Roodman, 2009). Por iso, nos modelos que non introducen a variable dependente retardada (modelo 2), replicáronse as estimacións empregando modelos estáticos de efectos fixos. En xeral, os resultados obtidos coinciden cos acadados previamente para os modelos dinámicos (GMM).

\section{Conclusións}

O obxectivo deste artigo tratou de investigar a validez da lei de Gibrat para unha mostra de 25.179 empresas ecuatorianas durante o período 2000-2013. Conforme a esta ley, a taxa de crecemento das empresas é independente do seu tamaño. Para realizar a contrastación empírica utilizouse o método xeneralizado dos momentos (GMM), co propósito de superar os problemas non observados dos efectos fixos, a persistencia e a endoxeneidade. Do cal se puido evidenciar que a mostra total das empresas non concordan cos supostos da lei de Gibrat, xa que as empresas máis pequenas creceron a un ritmo maior cás grandes, o que amosa que a taxa de crecemento depende do tamaño da empresa.

En canto á relación da idade sobre o crecemento cómpre destacar que existe unha relación non linear. As empresas nas súas primeiras etapas de vida posúen unha relación negativa co crecemento, porén, a unha determinada idade esta relación cambia a positiva. 0 efecto inicial da idade pode estar relacionado coas desvantaxes que enfrontan as empresas novas que é coñecida como "responsabilidade da novidade" (Coad, 2017). 0 asesoramento e apoio por parte das entidades gobernamentais debe ser máis intenso nas primeiras etapas de vida das empresas.

No que respecta á innovación non presenta ningunha relación significativa sobre o crecemento empresarial, é posible que os procesos de innovación estean relacionados con tempos de desenvolvemento máis longos ou aumentos no tempo, conforme argumentan Capasso et al. (2015). Porén, é necesario ter en conta que a innovación é unha estratexia moi incerta debido á incerteza con respecto a: os retornos da innovación, o éxito comercial, e a ameaza de apropiación e imitación (Coad et al., 2016).

Namentres que utilizar algún nivel de tecnoloxía presenta resultados non concluíntes, é evidente que se deben xerar procesos de avaliación da tecnoloxía utilizada nas empresas para establecer mecanismos de renovación ou capacitación a fin de que operar nalgún sector tecnolóxico se converta nunha fortaleza para a empresa. É fundamental ter en conta que o desenvolvemento tecnolóxico require interaccións dinámicas entre o coñecemento tácito e o explícito, onde o coñecemento individual e o grupal se transforman en produtos e tecnoloxía (Zahra et al., 2007).

Finalmente, o endebedamento débese orientar a financiar estratexias que permitan fortalecer os procesos de crecemento da empresa, para o cal cómpre ter un axeitado endebedamento orientado a financiar a sustentabilidade do crecemento, en base a unha redución do risco financeiro e custos da débeda (Aissa e Goaied, 2016).

Entre as limitacións, pódese mencionar que a mostra final está relacionada co alcance do estudo, que se circunscribe á análise das empresas de formación xurídica sociedade 
anónima e sociedade limitada. Adicionalmente a análise non considera factores específicos da industria ou taxas de concentración das empresas. Como consecuencia, un dos temas que no futuro se pode investigar é a converxencia da lei de Gibrat a longo prazo como formula Fiala (2017) ou analizar a relación cadrática do tamaño, para descubrir as relacións subxacentes, como desenvolveu Miralles-Quiros et al. (2017). En todo caso, as descobertas desta investigación permiten cubrir o baleiro na literatura empírica a nivel de Latinoamérica e contribúe a establecer pautas para os entes gobernamentais dos países que se atopan en desenvolvemento a fin de mellorar as políticas públicas das empresas.

\section{Bibliografía}

ADAMOU, A. \& SASIDHARAN, S. (2007). The impact of R\&D and FDI on firm growth in emergingdeveloping countries: Evidence from Indian manufacturing industries. Recuperado de https://ssrn.com/abstract=987024 or http://dx.doi.org/10.21 39/ssrn.987024.

AISSA, S. B. \& GOAIED, M. (2016). Determinants of Tunisian hotel profitability: The role of managerial efficiency. Tourism Management, 52, 478-487.

ALMSAFIR, M. K., NASSAR, I. A., AL-MAHROUQ, M. H., \& HAYAJNEH, J. A. (2015). The Validity of Gibrat's Law: Evidence from the Service Sector in Jordan. Procedia Economics and Finance, 23, 1602-1606.

ARELLANO, M. \& BOND, S. (1991). Some tests of specification for panel data: Monte Carlo evidence and an application to employment equations. The Review of Economic Studies, 58(2), 277-297.

ARÉVALO LUNA, G. A. (2014). Ecuador: economía y política de la revolución ciudadana, evaluación preliminar. Revista Apuntes del Cenes, 33(58), 109-134.

ASLAN, A. (2008). Testing Gibrat's law: Empirical evidence from panel unit root tests of Turkish firms.

ATUESTA, B., CUEVAS, F., \& ZAMBONINO, D. (2014). ¿Qué impulso la reducción de la pobreza y la desigualdad en Ecuador en la decada pasada? Una historia del mercado laboral urbano.

AUDRETSCH, D., KLOMP, L., SANTARELLI, E. \& THURIK, A. (2004). Gibrat's law: Are the services different? Review of Industrial Organization, 24, 301-324.

BENTZEN, J., MADSEN, E. S. \& SMITH, V. (2012). Do firms' growth rates depend on firm size? Small Business Economics, 39(4), 937-947.

BOND, S., (2002). Dynamic panel data models: a guide to micro data methods and practice. Portuguese Economic Journal, 1, 141-162.

CAINELLI, G., EVANGELISTA, R. \& SAVONA, M. (2006). Innovation and economic performance in services: a firm-level analysis, Cambridge Journal of Economics, 30(3), 435-458.

CAPASSO, M., TREIBICH, T., \& VERSPAGEN, B. (2015). The medium-term effect of R\&D on firm growth. Small Business Economics, 45 (1), 39-62.

CARRIZOSA, M. T. (2007). Firm growth, persistence and multiplicity of equilibria: an analysis of Spanish manufacturing and service industries. Tesis doctoral (Doctoral dissertation, Universitat Rovira i Virgili). Recuperado de http://www.tdx.cat/handle/10803/8447.

CEPAL, N. (2015). Financiamiento para el desarrollo en América Latina y el Caribe: un análisis estratégico desde la perspectiva de los países de renta media.

CHOI, B. P. (2010). The US property and liability insurance industry: Firm growth, size, and age. Risk Management and Insurance Review, 13(2), 207-224.

COAD, A. \& RAO, R. (2011). The firm-level employment effects of innovations in high-tech US manufacturing industries. Journal of Evolutionary Economics, 21(2), 255-283.

COAD, A. (2017). Firm age: a survey. Journal of Evolutionary Economics, 1-31.

COAD, A., SEGARRA, A. \& TERUEL, M. (2016). Innovation and firm growth: Does firm age play a role? Research Policy, 45(2), 387-400. 
COLOMBELLI, A., HANED, N. \& LE BAS, C. (2013). On firm growth and innovation: Some new empirical perspectives using French CIS (1992-2004). Structural Change and Economic Dynamics, 26, 14-26.

DAUNFELDT, S. O., \& ELERT, N. (2013). When is Gibrat's law a law?. Small Business Economics, 41(1), 133-147.

DAZA IZQUIERDO, J. (2016). La Ley de Gibrat en las economías emergentes: el caso de Brasil.

DE HAAN, J., SCHOLTENS, B., \& SHEHZAD, C. T. (2009). Growth and earnings persistence in banking firms: A dynamic panel investigation.

DEL MONTE, A. \& PAPAGNI, E. (2003). R\&D and the growth of firms: empirical analysis of a panel of Italian firms, Research policy, 32(6), 1003-1014.

FARIÑAS, J. C. \& MORENO, L. (1997). Size, age and growth: an application to Spanish manufacturing firms. En Documento de Trabajo 9705, Fundación Empresa Pública. Recuperado de $\mathrm{ftp}: / / \mathrm{ftp}$.fundacionsepi.es/pie/dt9705.pdf, consultado en marzo 09 de 2016.

FERNHOLZ, R. \& KOCH, C. (2016). Why are big banks getting bigger? Research Department, SSRN Working paper, Federal Reserve Banks of Dallas. [consultado 26 feb 2018] Disponible en: http://www.ricardofernholz.com/bankSize.pdf

FIALA, R. (2017). Testing Convergence toward Gibrat's Law for Czech Manufacturing Firms. Ekonomicky Casopis, 65(8), 737-750.

FOTOPOULOS, G., \& GIOTOPOULOS, I. (2010). Gibrat's law and persistence of growth in Greek manufacturing. Small Business Economics, 35(2), 191-202.

FUJIWARA, Y., DI GUILMI, C., AOYAMA, H., GALLEGATI, M., \& SOUMA, W. (2004). Do Pareto-Zipf and Gibrat laws hold true? An analysis with European firms. Physica A: Statistical Mechanics and its Applications, 335(1-2), 197-216.

GARCÍA-MANJÓN, J. \& ROMERO-MERINO, M. (2012). Research, development, and firm growth: Empirical evidence from European top R\&D spending firms. Research Policy, 41, 1084-1092.

GIBRAT, R. (1931), Les inégalités économiques. Recueil Sirey.

GODDARD, J., WILSON, J. \& BLANDON, P. (2002). Panel tests of Gibrat's Law for Japanese manufacturing. International Journal Industry Organization, 20(3), :415-433.

HARKATI, R., \& MOHAMAD, A. (2016). Gibrat's law and liquidity constraints: evidence from Malaysia industrial sector companies. International Journal of Economics, Management and Accounting, 24(2), 135.

HOMMA, T., TSUTSUI, Y., \& UCHIDA, H. (2014). Firm growth and efficiency in the banking industry: A new test of the efficient structure hypothesis. Journal of Banking \& Finance, 40, 143-153.

HOXHA, D. (2008). Entrepreneurship, employment and fast-growing firms in Kosova. World Review of Entrepreneurship, Management and Sustainable Development, 4(2-3), 203-217.

LABRA, R. \& TORRECILLAS, C. (2014). Guía CERO para datos de panel. Un enfoque práctico. UAMAccenture Working Papers, 16, 1-57.

LEITAO, J., SERRASQUEIRO, Z., \& NUNES, P. (2010). Testing Gibrat's Law for Listed Portuguese Companies: A Quantile Approach. International Research Journal of Finance and Economics, 37(1), 147-158.

LOI, T. H. \& KHAN, A. A. (2012). Determinants of firm growth: evidence from Belgian companies. Tesis de Máster, Universidad de Gante.

LOTTI, F., SANTARELLI, E. \& VIVARELLI, M. (2009). Defending Gibrat's Law as a long-run regularity. Small Business Economics, 32(1), 31-44.

LOTTI, F., SANTARELLI, E., \& VIVARELLI, M. (2003). Does Gibrat's Law hold among young, small firms?. Journal of evolutionary economics, 13(3), 213-235.

MANSFIELD, E. (1962). Entry, Gibrat's law, innovation, and the growth of firms. The American economic review, 52(5), 1023-1051.

MIRALLES-QUIROS, M., MILLARES-QUIROS, J. L., \& DAZA-IZQUIERDO, J. (2017). Contraste de la ley de Gibrat en la banca comercial brasileña. Contaduría y Administración, 62(5), 1643-1656. 
MUKHOPADHYAY, A., \& AMIRKHALKHALI, S. (2010). Profitability performance and firm size-growth relationship. Journal of Business \& Economics Research, 8(9), 121.

NASSAR, I. A., ALMSAFIR, M. K., \& AL-MAHROUQ, M. H. (2014). The Validity of Gibrat's Law in Developed and Developing Countries (2008-2013): Comparison based Assessment. ProcediaSocial and Behavioral Sciences, 129, 266-273.

NEIRA BURNEO, S. (2016). Inclusión financiera de las pymes en el Ecuador, Naciones Unidas, Santiago de Chile.

NUNES, P. M., GONÇALVES, M. \& SERRASQUEIRO, Z. (2013). The influence of age on SMEs' growth determinants: Empirical evidence. Small Business Economics, 40(2), 249-272.

PAGANO, P. \& SCHIVARDI, F. (2003). Firm size distribution and growth. The Scandinavian Journal of Economics, 105(2), 255-274.

PAVÓN, L. (2016). Inclusión financiera de las pymes en el Ecuador y México.

PIERGIOVANNI, R. (2010). Gibrat's Law in the "Third Italy": Firm Growth in the Veneto Region. Growth and Change, 41(1), 28-58.

RODRÍGUEZ-GULÍAS, M. J., FERNÁNDEZ-LÓPEZ, S. \& RODEIRO-PAZOS, D. (2016). Growth determinants in entrepreneurship: A longitudinal study of Spanish technology-based university spin-offs. Journal of International Entrepreneurship, 14(3), 323-344.

ROODMAN, D. (2009). How to do xtabond2: An introduction to difference and system GMM in Stata. Stata Journal, 9(1), 86-136.

SANTANA, F. B., VELÁZQUEZ, J. A. D. \& MARTEL, M. C. V. (2006). Concepto, perspectivas y medida del crecimiento empresarial. Cuadernos de administración, 19(31), 165-195.

SERRASQUEIRO, Z. \& NUNES, P. M. (2010). Non-linear relationships between growth opportunities and debt: Evidence from quoted Portuguese companies. Journal of Business Research, 63(8), 870-878.

SIMBAÑA, L. E., RODRÍGUEZ-GULÍAS, M. J., \& RODEIRO-PAZOS, D. (2017). Growth determinants in Ecuadorian companies. European Journal of Applied Business and Management, 3(3).

STULZ, R. (1990). Managerial discretion and optimal financing policies. Journal of financial Economics, 26(1), 3-27.

TANG, A. (2015). Does Gibrat's law hold for Swedish energy firms?. Empirical Economics, 49(2), 659674.

ZAHRA, S. A., VAN DE VELDE, E., \& LARRANETA, B. (2007). Knowledge conversion capability and the performance of corporate and university spin-offs. Industrial and Corporate Change, 16(4), 569608.

Julio Daza Izquierdo (2015). La ley de Gibrat en las economías emergentes: el caso de Brasil. Tesis Doctoral Unex. Disponible en http://dehesa.unex.es/bitstream/handle/10662/3986/TDUEX 2015 Daza Izquierdo. pdf? sequence $=1 \&$ is Allowed $=y$

Revista Galega de Economía: http://www.usc.es/econo/RGE/benvidag.htm 